Iranian Journal of Pathology | ISSN: 2345-3656

\title{
The Correlation BetweenSerum Vitamin D Level and Total Antioxidant Capacity in Diabetic and Non-diabetic Subjectsin Iran
}

\author{
Ali Zare-Mirzaie', Behrang Kazeminezhad ${ }^{2 *}$, Mona Akbari Ghouchani ${ }^{1}$ \\ 1. Dept of Pathology, Iran University of Medical Sciences, Rassool Akram Hospital, Tehran, Iran \\ 2. Dept of Pathology, Shahid Beheshti University of Medical Science, Shahid Modarres Hospital, Tehran, Iran
}

\begin{tabular}{c}
\hline KEYWORDS \\
\hline TotalAntioxidant Capacity; \\
Diabetes Mellitus; \\
Oxidative Stress; \\
Vitamin D \\
\hline
\end{tabular}

Article Info

Received 08 April 2017; Accepted 12 Des 2017; Published Online 17 July 2018;

\section{ABSTRACT}

Background \& objective: Increase in intra- and extracellular glucose levels can cause oxidative stress, and theprolonged imbalance between prooxidants and antioxidantscan lead to cell damage and the associated complications in patients with diabetes. Vitamin D acts as a strong antioxidant in the body and several studies emphasized on its important role to preventoxidative stress in prediabeticand diabetic subjects. The current study aimed at determining and comparingthe total antioxidant capacity (TAC) in individuals with hemoglobin A1c(HbA1c) below and above 6.5\%, and its correlation with vitamin D levels.

Methods: The current cross sectional study was conducted on a total of 107 patients with diabetes (HbA1c $>6.5 \%)$ and 107 non-diabetic subjects (HbA1c $<6.5 \%)$ referred toRassoolAkram Hospital, Tehran, Iranfrom 2015 to 2016, as the sample population. The two groups were compared regarding their TAC and vitamin D serum levels and the association between vitamin D concentration and TAC was evaluated.

Results: Age and body mass index (BMI)were significantly higher in patients with diabetes,comparedwiththe serum levels of vitamin D and TAC $(\mathrm{P}<0.001$ for both). Both TAC and vitamin D levels were significantly lower in the group with diabetesand a weak significant correlation was observedbetween the two factors $(\mathrm{r}=0.2, P=0.003)$. In multivariate regression model, the duration of diabetes was also significantly associated with TAC level (beta coefficient $=-0.82, P<0.001$ ).

Conclusion: The low serum levels of TAC and vitamin D in patients with diabetescould be indicative of oxidative stress in the presence of high blood glucose levels. Supplementation of vitamin D in patients with diabetes might be effective to control thenegative impacts of the disease and decreasecells' exposure to oxidative environment in prediabetes.

Corresponding information:

Behrang Kazeminezhad, Dept of Pathology, ShahidBeheshti University of Medical Science,

Shahid Modarres Hospital, Tehran, Iran. E-mail: dkazeminezhad@gmail.com

Copyright (C) 2018, IRANIAN JOURNAL OF PATHOLOGY. This is an open-access article distributed under the terms of the Creative Commons Attribution-noncommercial 4.0 International License which permits copy and redistribute the material just in noncommercial usages, provided the original work is properly cited.

\section{Introduction}

Free radicals are the reactive molecules produced during metabolic processes in the human body. High levels of free radicals can lead to destruction of proteins, lipid membrane and nucleic acids, and eventually cell death. These unstable molecules are implicated in the pathogenesis of various chronic diseases such as atherosclerosis, myocardial insufficiency, autoimmune diseases, and type 2 diabetes (1). Oxidative stress markers in tissue injury and antioxidant systems include superoxide dismutase (SOD), gluta- thione peroxidase (GPx), glutathione reductase (GR), catalase, ceruloplasmin, metallothionein proteins, etc. (2).

Various methods can be used to evaluate the antioxidant status through measuring total antioxidant capacity (TAC). The major advantage of this method is that instead of only measuring the level of one antioxidant, all antioxidants in a biological specimen are measured simultaneously. Accordingly, TAC is used as a new biomarker for diagnosis, prognosis, and prevention of many diseases including diabetes 
(3). Therefore, measuring TAC as an indicator of all oxidative stress agents and the defensive effects of antioxidants can be used as the first step to evaluate a patients' health status (4).

Different mechanisms are proposed to increase intra- and extracellular glucose concentrations and the resultant oxidative stress including glucose auto-oxidation, glucose non-enzymatic glycosylation, and increase in the level of glucose derived from the final product of advanced glycosylation. Evidence suggests that oxidative stress due to hyperglycemia presents before the development of diabetes complications (5).

Type 2 diabetes is a chronic disease that significantly affects the patients' quality of life and considering the prolonged course of the disease and its complications and the fact that they could be prevented, early diagnosis in patients with risk factors seems to be of utmost importance. Hence, routine screening for diabetes starts at age 45 years and is repeated at least every three years, while in subjects at risk it is started earlier $(6,7)$.

American Diabetes Association (ADA) is always trying to update its guidelines in order to diagnose patients with diabetes earlier with a higher accuracy. According to their latest guidelines, type 2 diabetes is defined as hemoglobin A1c (HbAlc) $\geq 6.5 \%$, fasting blood sugar (FBS) $\geq 126 \mathrm{mg} / \mathrm{dL}$, or a 2 -hour post prandial glucose $\geq 200 \mathrm{mg} / \mathrm{dL}$ after $75 \mathrm{~g}$ oral glucose intake $(8,9)$.

Recently, extensive research is conducted on different roles of vitamin D and it is found to have various roles other than the previously known function in the metabolism of skeletal systems and regulation of calcium and phosphorus ions, including neuromuscular function, cancer prevention, and risk reduction in cardiovascular diseases, hypertension, dyslipidemia, and diabetes $(1,10,11)$. Also, vitamin $\mathrm{D}$ is attributed to prevention of chronic diseases such as diabetes, cardiovascular diseases (CVD), and chronic kidney disease (CKD) by regulation of oxidative stress through the following ways: it induces the expression of several molecules involved in the antioxidant defense system including glutathione (GSH), GSH peroxi- dase, and superoxide dismutase (SOD), and suppresses the expression of NADPH (nicotinamide adenine dinucleotide phosphate) oxidase (12).

Studies show that a decrease in serum vitamin D lev$\mathrm{el}$ is associated with an increase in FBS, decrease in sensitivity to insulin, and increased risk of type 2 diabetes (13) and it can affect blood glucose control and oxidative stress in patients with diabetes (14). A direct correlation was also found between serum vitamin D levels and the TAC (15). This vitamin can protect the cell membrane by inhibiting lipid peroxidation and exert its antioxidant effects through changing antioxidant enzymes (16-18). It acts as a scavenger of free radicals in the early stages, before activation of pathways against oxidative stress $(1,10,11,16,19-21)$. Accordingly, the current study aimed at comparing the serum vitamin D and TAC levels in non-diabetic and diabetic subjects and evaluating the correlation between the two.

\section{Materials and Methods}

\section{Study subjects}

The current analytical, cross sectional study was conducted on 214 subjects aged above 18 years including 107 patients with diabetes ( $\mathrm{HbA} 1 \mathrm{c}>6.5 \%)$ and 107 non-diabetic subjects $(\mathrm{HbA} 1 \mathrm{c}<6.5 \%)$, referred to Central Laboratory of Rassool Akram Hospital from 2015 to 2016 with a request of vitamin D serum level measurement as the sample population. The study objectives were fully explained to the patients and after obtaining their consent, the study was performed. The exclusion criteria were CKD, thyroid diseases (hyperand hypothyroidism), liver failure, pregnancy, and breastfeeding, specific nutritional regimens, alcohol consumption, cigarette smoking, blood donation in the last six months and a positive history for taking lipid reduction pharmaceutical agents, anticonvulsants, vitamin D or calcium supplements, and any other medications affecting bone metabolism.

\section{Sample collection}

After eight hours of fasting overnight, $10 \mathrm{~mL}$ of venous blood was collected from the participants. Serum was separated from the coagulated blood in the 
tubes via centrifugation at $2000 \mathrm{rpm}$ for 15 minutes at $4^{\circ} \mathrm{C}$. Samples were then transferred into $2 \mathrm{~mL}$ specific microtubes and stored at $-20^{\circ} \mathrm{C}$.

\section{Laboratory analysis}

TAC was measured using the ferric reducing antioxidant power (FRAP) assay (ZellBio ELISA Kit, Germany), 25-hydroxyvitamin D was measured by the Euroimmun ELISA (the enzyme-linked immunosorbent assay) kit (Germany) and the serum concentrations of FBS, uric acid, albumin, and total bilirubin were assessed using the AutoAnalyzer device BT3000 via Pars Azmoon ELISA kits( Pars Azmoon Co., Tehran, Iran).

\section{Statistical analysis}

Qualitative and quantitative variables were compared using the Chi-Square test and the Student t test, respectively. Correlation between the main variables of the study was assessed based on the Pearson correlation coefficient. Since TAC is significantly correlated with uric acid levels, calculated total antioxidant status (cTAS) was also computed based on the formula presented by Anvari et al., (10) and compared between the two groups:

cTAS $=(0.63 \times$ albumin $)+(1.02 \times$ uric acid $)+(1.53 \times$ bilirubin)

Eventually, linear regression analysis was performed to control the effects of confounding factors. Results were presented as regression coefficient and 95\% confidence interval (CI) for the main variables. All the analyses were performed with SPSS version 20 (Chicago, Illinois, SPSS Inc.), considering a P-value of $<0.05$ as statistically significant.

\section{Results}

The sample population comprised of 107 patients with diabetes (42.1\% male) and 107 non-diabetic subjects $(29.9 \%$ male). In order to assess the agreement between the two ELISA kits used to measure TAC, the serum samples of four patients were evaluated by both kits and the results showed no significant difference between the two $(P=0.789)$.

Table 1. Demographic Characteristics and Laboratory Results in the Two Groups of Diabetics and Non-diabetic Subject

\begin{tabular}{|c|c|c|c|}
\hline Characteristic & Non-diabetic & Diabetic & P-value \\
\hline Age ( yr) & $49.0 \pm 15.1$ & $58.94 \pm 11.7$ & $<0.001$ \\
\hline Male gender, n (\%) & $32(29.9)$ & $45(42.1)$ & 0.064 \\
\hline BMI( $\left.\mathrm{kg} / \mathrm{m}^{2}\right)$ & $26.0 \pm 5.3$ & $28.1 \pm 5.2$ & 0.004 \\
\hline HbA1c (\%) & $5.5 \pm 0.5$ & $7.8 \pm 1.5$ & $<0.001$ \\
\hline Fasting blood sugar (mg/dL) & $98.2 \pm 12.3$ & $180.4 \pm 70.3$ & $<0.001$ \\
\hline Vitamin D (ng/mL) & $15.67 \pm 6.3$ & $9.6 \pm 5.3$ & $<0.001$ \\
\hline $\mathrm{TAC}(\mu \mathrm{m} / \mathrm{L})$ & $448.3 \pm 112.8$ & $339.1 \pm 47.9$ & $<0.001$ \\
\hline Uric acid (mg/dL) & $5.5 \pm 1.5$ & $5.0 \pm 1.2$ & 0.035 \\
\hline Albumin (mg/dL) & $4.0 \pm 0.4$ & $2.8 \pm 0.71$ & $<0.001$ \\
\hline Total bilirubin (mg/dL) & $0.88 \pm 0.38$ & $0.62 \pm 0.16$ & 0.008 \\
\hline $\operatorname{cTAS}(\mu \mathrm{M} / \mathbf{L})$ & $9.6 \pm 1.8$ & $7.2 \pm 1.2$ & $<0.001$ \\
\hline
\end{tabular}


Table 1 presents the differences between the two groups of non-diabetic and diabetic subjects regarding their demographic characteristics and laboratory results. Accordingly, age, body mass index (BMI), FBS, and HbA1c were significantly higher in the diabetics group, while their levels of vitamin D (Figure 1), TAC (Figure 2), cTAS, uric acid, total bilirubin, and albumin were significantly lower than those of the control group.

Correlation between TAC levels with the main variables of the study was assessed and the results are presented in Table 2.
As can be observed, TAC level was negatively correlated with age $(\mathrm{r}=-0.20, P=0.003)$, FBS ( $\mathrm{r}=-0.38$, $P<0.001)$, and duration of diabetes ( $\mathrm{r}=0.2, P=0.003$ ). The correlation between TAC level and vitamin D concentration, which was a weak and positive relationship, although statistically significant $(\mathrm{r}=0.2$, $P=0.003$ ) (Figure 3).

Multivariate linear regression analysis was used to assess the effects of confounding factors on the correlation between vitamin D level and TAC. Due to the heteroscedasticity, TAC logarithm was used in this analysis. Confounding factors in the model included age, $\mathrm{HbA1c}$, and duration of diabetes.

Table 2. Correlation Between TAC Level and the Main Variables Assessed in the Study

\begin{tabular}{l|c|c}
\hline & Characteristic & \multicolumn{2}{c}{ TAC } & P-value \\
\cline { 2 - 3 } Age & r & 0.003 \\
BMI & -0.20 & 0.145 \\
FBS & 0.10 & $<0.001$ \\
HbA1c & -0.37 & $<0.001$ \\
\hline Duration of diabetes & -0.40 & $<0.001$ \\
\hline Vitamin D & -0.66 & 0.003 \\
\hline
\end{tabular}

BMI: body mass index; FBS: fasting blood sugar; HbA1c: hemoglobin A1c;

TAC: total antioxidant capacity

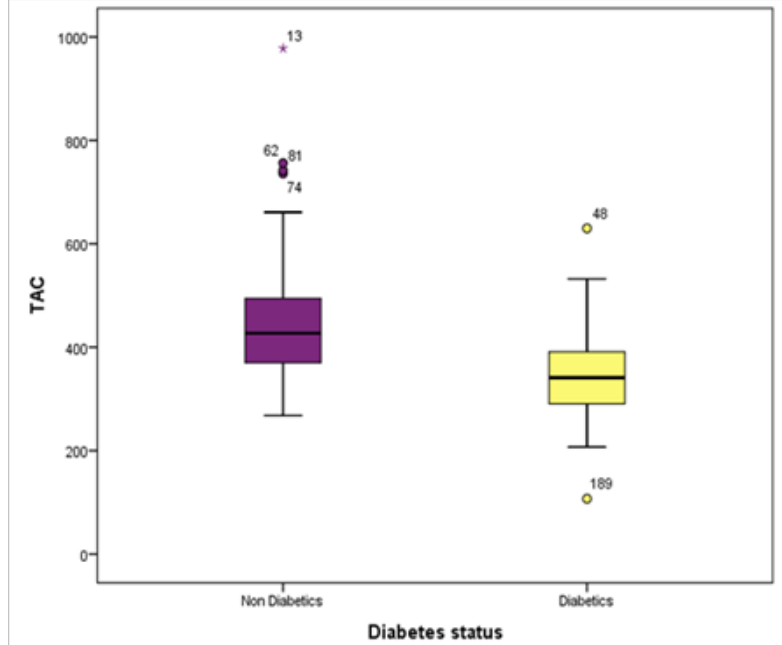

Figure 1. The Difference in TAC Level Between the Two Groups of Diabetic and Non-diabetic Subjects

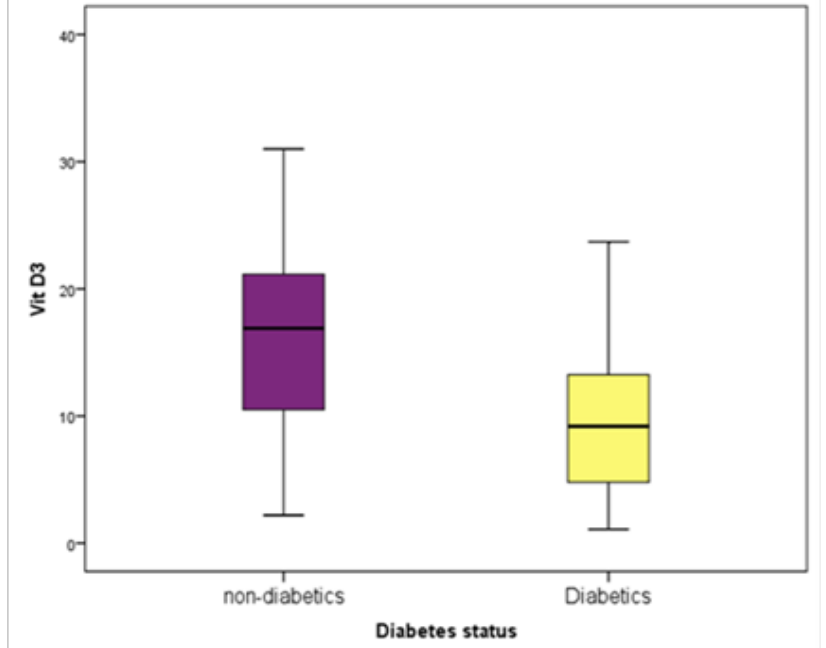

Figure 2. The Difference in Vitamin D Level Between the Two Groups of Diabetic and Non-diabetic Subjects 


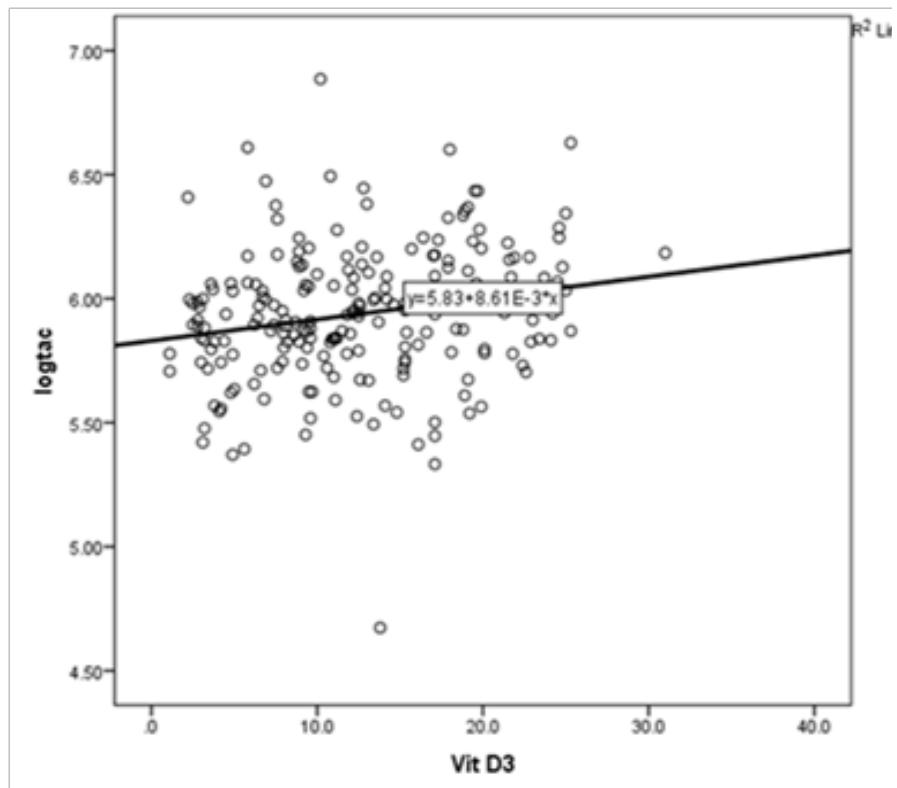

Figure 3. Correlation Between TAC and Vitamin D Levels

Table 3. Multivariate Linear Regression Analysis on the Level of TAC

\begin{tabular}{|c|c|c|c|}
\hline Variable & Beta Coefficient & $95 \%$ CI & P-value \\
\hline Age & 0.02 & $-0.001,0.001$ & 0.680 \\
\hline HbA1c & 0.079 & $-0.003,0.015$ & 0.204 \\
\hline Duration of diabetes & -0.82 & $-0.026,-0.019$ & $<0.001$ \\
\hline Vit D & -0.06 & $-0.003,0.001$ & 0.267 \\
\hline
\end{tabular}

Based on the results of this analysis presented in Table 3, a negative significant correlation was observed between the duration of diabetes and the TAC level $(P<0.001)$ and the differences in the logarithm of their TAC levels remained significant between the two groups.

\section{Discussion}

Considering the importance of controlling free radicals in progression of diabetes complications and the fact that vitamin D might have played a role to protect the pathways, the current study aimed at comparing the level of vitamin D and TAC in the two groups of diabetic and non-diabetic subjects and assessing the correlation between the two factors.

Based on the results of the current study, both TAC and vitamin D levels were significantly lower in the diabetic group and a weak significant correlation was observed between the two factors. In the multivariate regression model employed in the current study, the duration of diabetes was also significantly associated with TAC.

In previous studies, the rate of vitamin D deficiency in diabetic and non-diabetic subjects was reported $82 \%$ and $75 \%$ in the Iranian population, respectively (11). However, considering a vitamin D level of $<30$ $\mathrm{ng} / \mathrm{mL}$ in the current study, the rate of vitamin $\mathrm{D}$ deficiency and insufficiency was $99.5 \%$ in the whole sample population. This rate was $100 \%$ in the current study patients with diabetes and $91.9 \%$ among nondiabetic subjects. The higher prevalence of vitamin D deficiency and insufficiency could be attributed to the low sample size of patients evaluated in the current study, and the inclusion and exclusion criteria established for it. For instance, including subjects less than 18 years old or other groups that were excluded from the current study might have changed the overall calculated rate.

Possible explanations for the high rate of vitamin D deficiency in Iranian population are lack of exposure 
to sunlight, skin pigmentation, dietary habits including low daily vitamin D intake and probably polymorphism in the genes of vitamin D receptor (VDR), and vitamin D-binding protein (VDP) $(22,23)$.

In the study conducted by Saedisomeolia et al. (11), 100 patients with diabetes were compared with 100 healthy subjects regarding their serum levels of vitamin D, calcium, phosphorus, parathyroid hormone, glucose, HbA1c, and TAC. They reported that $82 \%$ of the patients with diabetes and $75 \%$ of healthy subjects had vitamin D deficiency and insufficiency. Vitamin D level was negatively correlated with glutathione reductase (GR) and glutathione peroxidase (GPx) activity and was positively associated with TAC level. These researchers mentioned that vitamin $\mathrm{D}$ could be effective in controlling blood glucose level and oxidative stress in patients with diabetes. Their findings were quite similar to the results of the current study, which were indicative of a weak correlation between vitamin D and TAC levels. The current study could also argue that vitamin D supplementation might be able to improve oxidative stress in patients with diabetes; however, such deduction can only be made through implementation of clinical trials on diabetic subject.

In another study by Saif-Elnasr et al. (21), vitamin D level and oxidative stress were compared between the patients with diabetes and the controls. The results showed a significantly lower level of vitamin $\mathrm{D}$ among subjects with diabetes $(P=0.015)$ compatible with the results of the current study. GPx activity was also significantly lower in this group ( $P=0.0480)$. They found no significant correlation between vitamin D level and GPx activity. The authors noted that vitamin D played an important role in the pathogenesis of type 2 diabetes and its supplementation can improve glycemic control and oxidative stress status in patients with diabetes. The fact that these researchers only evaluated the level of GPx activity and not the overall TAC, might be the reason they did not find a significant correlation.

Considering the significant correlation between serum uric acid level and TAC (24), its concentrat tion was also measured and compared between the two groups in the current study. The serum level of uric acid, total bilirubin, and albumin were all significantly lower in the group with diabetes compared with those of the control group. Accordingly, cTAS was also significantly lower in patients with diabetes congruent with previous studies showing that the antioxidant effects of uric acid were higher in the nondiabetic subjects (10).

Current evidence implicates various factors to develop oxidative stress and its severity in patients with diabetes. Identification of all these factors demands extensive research on patients with diabetes at different stages of their disease, taking into account the duration of diabetes, and presence and severity of its complications. The antioxidant capacity of the serum based on both TAC and cTAS can help to provide a better control of the disease in patients with diabetes, prevent long-term complications, and even predict development of the disease in subjects at risk through measuring oxidative stress-related metabolites in the cells exposed to high blood glucose levels; although the evidence of the significant role of vitamin D on inflammation, insulin resistance, and insulin secretion in the pathogenesis of obesity, metabolic syndrome, and type 2 diabetes, its potential function in treatment and prevention of type 2 diabetes mellitus is unclear (25).

The current study was conducted as a cross sectional survey, which could be pointed out as one of its main limitations, since it cannot provide evidence on the direct effects of vitamin D supplementation on TAC level. The fact that the current study sample population only included Iranian people was another limitation, which subjects the results of the study to selection bias and the effects of race and nationality.

\section{Conclusion}

Overall, the findings of the current study showed a lower TAC in patients with diabetes, which could be attributed to the presence of oxidative stress in the studied subjects. The serum level of vitamin D was also lower in patients with diabetes, which considering the antioxidant effects of this vitamin can further 
worsen oxidative stress in patients with diabetes. In this regard, supplementation of vitamin D in patients with diabetes might be effective to control negative impacts of the disease and decrease cells' exposure to oxidative environment in prediabetes. Nevertheless, further investigations are required to precisely determine the pathogenesis of oxidative stress in patients with diabetes and its associated factors. It is strongly recommended that clinical trials be conducted to assess the reversibility of the oxidative processes in such patients.

\section{Acknowledgment}

The authors gratefully acknowledge the financial support of oncopathology research center of Iran university of medical sciences.

\section{Conflict of interests}

The authors declared no conflict of interest.

\section{References}

1. Bonnefont-Rousselot D, Bastard J, Jaudon M, Delattre J. Consequences of the diabetic status on the oxidant/antioxidant balance. Diabetes Metab. 2000;26(3):163-77. PMID:10880889

2. Hamden K, Carreau S, Jamoussi K, Miladi S, Lajmi S, Aloulou D, et al. 1 $\alpha, 25$ dihydroxyvitamin D3: therapeutic and preventive effects against oxidative stress, hepatic, pancreatic and renal injury in alloxan-induced diabetes in rats. J Nutr Sci Vitaminol (Tokyo). 2009;55(3):215-22. https://doi.org/10.3177/ insv.55.215 PMID: 19602829

3. Kusano C, Ferrari B. Total antioxidant capacity: a biomarker in biomedical and nutritional studies. J Cell Mol Biol. 2008;7(1):1-15. jcmb. halic.edu.tr/pdf/7-1/BOLUM_1.pdf

4. Deeb KK, Trump DL, Johnson CS. Vitamin D signalling pathways in cancer: potential for anticancer therapeutics. Nat Rev Cancer. 2007;7(9):684-700. h https://doi.org/10.1038/ nrc2196 PMID:17721433

5. Deluca HF, Cantorna MT. Vitamin D: its role and uses in immunology. FASEB J. 2001;15(14):2579-85. https://doi.org/10.1096/ fj.01-0433rev PMID:11726533

6. Penn L, White M, Lindström J, den Boer AT,
Blaak E, Eriksson JG, et al. Importance of weight loss maintenance and risk prediction in the prevention of type 2 diabetes: analysis of European Diabetes Prevention Study RCT. PLoS One. 2013;8(2):e57143. https://doi. org/10.1371/journal.pone.0057143 PMID:23451166 PMCID:PMC3581561

7. Vaz JA, Patnaik A. Diabetes mellitus: Exploring the challenges in the drug development process. Perspect Clin Res. 2012;3(3):109-12. https://doi. org/10.4103/2229-3485.100660 PMID:23125962 PMCID:PMC3487225

8. American Diabetes Association. (2) Classification and diagnosis of diabetes. Diabetes Care. 2015;38(38S):S8-16. https://doi.org/10.2337/ dc15-S005 PMID:25537714

9. Čolak E, Dimitrijević-Srećković V, Djordjević PB, Stanković S, Glišić B, Srećković B, et al. Biomarkers of enzymatic and non-enzymatic antioxidative defense in type 2 diabetes mellitus-comparative analysis. Biochemia Medica. 2008;18(1):42-51. https://doi.org/10.11613/ BM.2008.006

10. Anvari MS, Babaki MM, Boroumand MA, Eslami B, Jalali A, Goodarzynejad H. Relationship between calculated total antioxidant status and atherosclerotic coronary artery disease. Anatol J Cardiol. 2016;16(9):689-95. https:// dx.doi.org/10.5152\%2FAnatolJCardiol.2015.6482 PMID:27488746 PMCID:PMC5331354

11. Saedisomeolia A, Taheri E, Djalali M, Djazayeri A, Qorbani M, Rajab A, Larijani B. Vitamin D status and its association with antioxidant profiles in diabetic patients: A cross-sectional study in Iran. Indian J Med Sci. 2013;67(1-2):29-37. PMID:24178339

12. Mokhtari Z, Hekmatdoost A, Nourian M. Antioxidant efficacy of vitamin D. J Parathyr Dis. 2017;5(1):11-6. http://www.jparathyroid.com/Abstract/JPD_20160924145139

13. Holick MF. Diabetes and the vitamin D connection. Curr Diab Rep. 2008;8(5):393-8. https://doi. org/10.1007/s11892-008-0068-0 PMID: 18778589

14. Gagnon C, Lu ZX, Magliano DJ, Dunstan DW, Shaw JE, Zimmet PZ, et al. Serum 25-hydroxyvitamin $\mathrm{D}$, calcium intake, and risk of 
219. The Correlation BetweenSerum Vitamin D ...

type 2 diabetes after 5 years. Diabetes Care. 2011;34(5):1133-8. https://doi.org/10.2337/dc102167 PMID:21430082 PMCID:PMC3114481

15. Riachy R, Vandewalle B, Kerr Conte J, Moerman E, Sacchetti P, Lukowiak B, et al. 1, 25-dihydroxyvitamin D3 protects RINm5F and human islet cells against cytokine-induced apoptosis: implication of the antiapoptotic protein A20. Endocrinology. 2002;143(12):4809-19. https:// doi.org/10.1210/en.2002-220449 PMID: 12446608

16. Bischoff-Ferrari HA, Giovannucci E, Willett WC, Dietrich T, Dawson-Hughes B. Estimation of optimal serum concentrations of 25-hydroxyvitamin D for multiple health outcomes. Am J Clin Nutr. 2006;84(1):18-28. https://doi. org/10.1093/ajcn/84.1.18 PMID: 16825677

17. Haghdoost A, Rezazadeh-Kermani M, Sadghi$\operatorname{rad} B$, Baradaran H. Prevalence of type 2 diabetes in the Islamic Republic of Iran: systematic review and meta-analysis. East Mediterr Health J. 2009;15(3):591-9. PMID:19731775

18. Kostoglou-Athanassiou I, Athanassiou P, Gkountouvas A, Kaldrymides P. Vitamin D and glycemic control in diabetes mellitus type 2 . Ther Adv Endocrinol Metab. 2013;4(4):122-

8. https://doi.org/10.1177/2042018813501189 PMID:23997931 PMCID:PMC3755528

19. Awumey EM, Mitra DA, Hollis BW, Kumar R, Bell NH. Vitamin D Metabolism Is Altered in Asian Indians in the Southern United States: A Clinical Research Center Study 1. J Clin Endocrinol Metab. 1998;83(1):169-73. https://doi. org/10.1210/jc.83.1.169 PMID: $\underline{9435436}$
20. Ceriello A. New insights on oxidative stress and diabetic complications may lead to a "causal" antioxidant therapy. Diabetes Care. 2003;26(5):1589-96. https://doi.org/10.2337/diacare.26.5.1589 $\quad$ PMID: 12716823

21. Saif-Elnasr M, Ibrahim IM, Alkady MM. Role of Vitamin D on glycemic control and oxidative stress in type 2 diabetes mellitus. J Res Med Sci. 2017;22(1):22. https://doi. org/10.4103/1735-1995.200278 PMID: $\underline{28413419}$ PMCID:PMC 5377965

22. Evans JL, Goldfine ID, Maddux BA, Grodsky GM. Oxidative stress and stress-activated signaling pathways: a unifying hypothesis of type 2 diabetes. Endocr Rev. 2002;23(5):599622. https://doi.org/10.1210/er.2001-0039 PMID: 12372842

23. Jolfaie NR, Rouhani MH, Onvani S, Azadbakht L. The association between Vitamin D and health outcomes in women: A review on the related evidence. J Res Med Sci. 2016;21:76. https://dx.doi.org/10.4103\%2F1735-1995.189693 PMID:27904621 PMCID:PMC5122178

24. Sautin YY, Johnson RJ. Uric acid: the oxidantantioxidant paradox. Nucleosides Nucleotides Nucleic Acids. 2008;27(6-7):608-19. https://doi. org/10.1080/15257770802138558 PMID: 18600514 PMCID:PMC2895915

25. Garbossa SG. Folli F. Vitamin D, sub-inflammation and insulin resistance. A window on a potential role for the interaction between bone and glucose metabolism. Rev Endocr Metab Disord. 2017;18(2):243-58. https://doi. org/10.1007/s11154-017-9423-2 PMID:28409320

\section{How to Cite This Article}

Zare-Mirzaie A, Kazeminezhad B, Akbari Ghouchani M. The Correlation Between Serum Vitamin D Level and Total Antioxidant Capacity in diabetic and Non-diabetic Subjects in Iran. Iranian Journal of Pathology, 2018; 13(2): 212-219. 